\title{
Digital nuclear shell model
}

\author{
Lutvo Kurić \\ Institute of Economics, University of Sarajevo, Trg Oslobođenja 1, Sarajevo, \\ Bosnia and Herzegovina \\ E-mail address: lutvokuric@yahoo.com
}

\begin{abstract}
The subject of this thesis is a digital approach to the investigation of the digital basis of digital nuclear shell model. The shell model is partly analogous to the atomic shell model which describes the arrangement of electrons in an atom, in that a filled shell results in greater stability. When adding nucleons to a nucleus, there are certain points where the binding energy of the next nucleon is significantly less than the last one. Magic numbers of nucleons: 2, 8, 20, 28, 50, 82, 126 which are more tightly bound than the next higher number, is the origin of the shell model. "In a threedimensional harmonic oscillator the total degeneracy at level $\mathrm{n}$ is $\frac{(n+1)(n+2)}{2}$. Due to the spin, the degeneracy is doubled and is $(n+1)(n+2)$. Thus the magic numbers would be $\sum_{n=0}^{k}(n+1)(n+2)=\frac{(k+1)(k+2)(k+3)}{3}$ for all integer $\mathrm{k}$. This gives the following magic numbers: $2,8,20,40,70,112 \ldots$, which agree with experiment only in the first three entries. These numbers are twice the tetrahedral numbers $(1,4,10,20,35,56 \ldots)$ from the Pascal Triangle". http://en.wikipedia.org/wiki/Nuclear_shell_model. The digital mechanism of shell model have been analyzed by the application of cybernetic methods, information theory and system theory, respectively. This paper is to report that we discovered new methods for development of the new technologies in nuclear physics and chemistry. It is about the most advanced digital technology which is based on program, cybernetics and informational systems and laws. The results in practical application of the new technology could be useful in physics, chemistry, bioinformatics, and other natural sciences.
\end{abstract}

Keywords: Nuclear physics; shell model; magic number of nucleons; Pascal Triangle

\section{INTRODUCTION}

The sequencing of the nuclear shell model is determined not only by distin chemical features, but also by cybernetic and information principles. For this reason, research in this field deals more with the quantitative rather than qualitative characteristcs of chemcal information and its chemical basis. For the purposes of this paper, specific physical and chemical factors have been selected in order to express the chemical information for shell model. Numerical values are them assigned to these factors, enabling them to be measured. In this way it is possible to determine oif a connection really exists between the quantitative ratios in the process of transfer of chemical information and the qualitative appearance of the shell model. To select these factors, preference is given to classical physical and chemical 
parameters, their analog values, and their frenquencies. There is a arge numbers of these parameters, and each of their gives important chemical information. Going through this process, it becomes clear that there is a mathematical relationship between quantitative ratios and the qualitative appearance of the digital nuclear shell model and that there is a measurement method that can be used to describe the chemistry of this model.

\section{RESULTS}

"In nuclear physics, a magic number is a number of nucleons (either protons or neutrons) such that they are arranged into complete shells within the atomic nucleus. The seven most widely recognized magic numbers as of 2007 are 2, 8, 20, 28, 50, 82, and 126 (sequence A018226 in OEIS). Atomic nuclei consisting of such a magic number of nucleons have a higher average binding energy per nucleon than one would expect based upon predictions such as the semi-empirical mass formula and are hence more stable against nuclear decay". (http://en.wikipedia.org/wiki/Magic_number_(physics)").

\section{Predicted magic numbers}

Toeplitz matrix $\{2,8,20,28,50,82,126\}$

\section{Input:}

ToeplitzMatrix $[\{2,8,20,28,50,82,126\}]$

\section{Result:}

$(2: 2)=1 ;(8: 2)=4 ;(20: 2)=10 ;$ etc.

$$
2\left(\begin{array}{ccccccc}
1 & 4 & 10 & 14 & 25 & 41 & 63 \\
4 & 1 & 4 & 10 & 14 & 25 & 41 \\
10 & 4 & 1 & 4 & 10 & 14 & 25 \\
14 & 10 & 4 & 1 & 4 & 10 & 14 \\
25 & 14 & 10 & 4 & 1 & 4 & 10 \\
41 & 25 & 14 & 10 & 4 & 1 & 4 \\
63 & 41 & 25 & 14 & 10 & 4 & 1
\end{array}\right)
$$

\section{Dimensions}


Matrix plot:

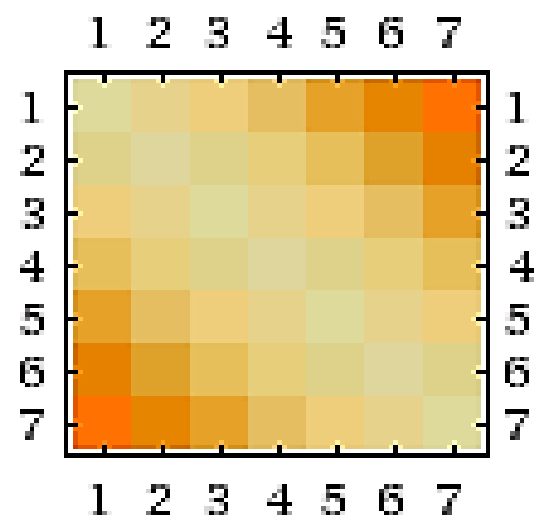

Determinant:

2151534827929600

$2151534827929600=46384640^{2}$

symmetric

toeplitz

\section{Determinant:}

$-724375168$

Trace:

14

\section{Characteristic polynomial:}

$-x^{7}+14 x^{6}+42260 x^{5}+1577672 x^{4}+18080960 x^{3}+46384640 x^{2}-259459776 x-$ 724375168

Eigenvalues:

$\lambda_{1} \approx 229.551$

$\lambda_{2} \approx-177.333$

$\lambda_{3} \approx-18.4947$

$\lambda_{4} \approx-10.5847$

$\lambda_{5} \approx-10.1218$

$\lambda_{6} \approx 3.52885$

$\lambda_{7} \approx-2.54493$ 


\section{Eigenvectors:}

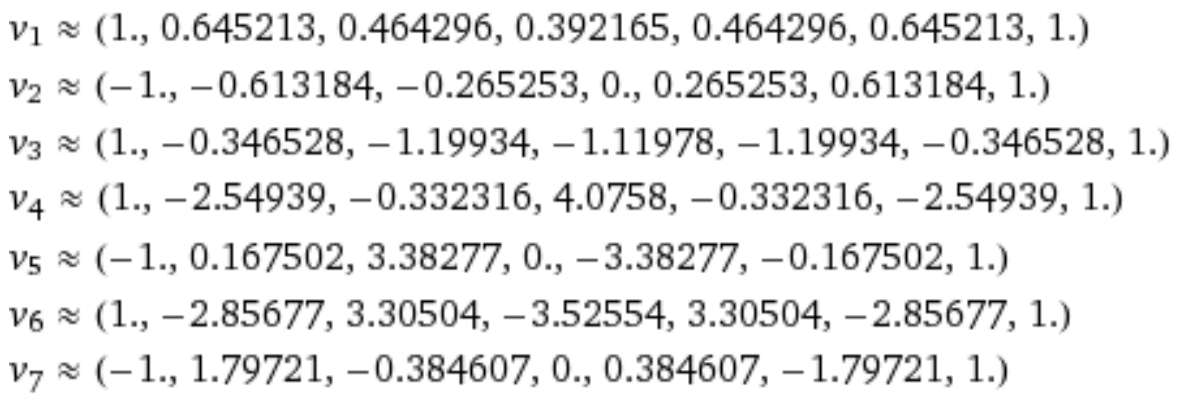

\section{Diagonalization:}

$M=S . J . S^{-1}$

where

$M=\left(\begin{array}{ccccccc}2 & 8 & 20 & 28 & 50 & 82 & 126 \\ 8 & 2 & 8 & 20 & 28 & 50 & 82 \\ 20 & 8 & 2 & 8 & 20 & 28 & 50 \\ 28 & 20 & 8 & 2 & 8 & 20 & 28 \\ 50 & 28 & 20 & 8 & 2 & 8 & 20 \\ 82 & 50 & 28 & 20 & 8 & 2 & 8 \\ 126 & 82 & 50 & 28 & 20 & 8 & 2\end{array}\right)$

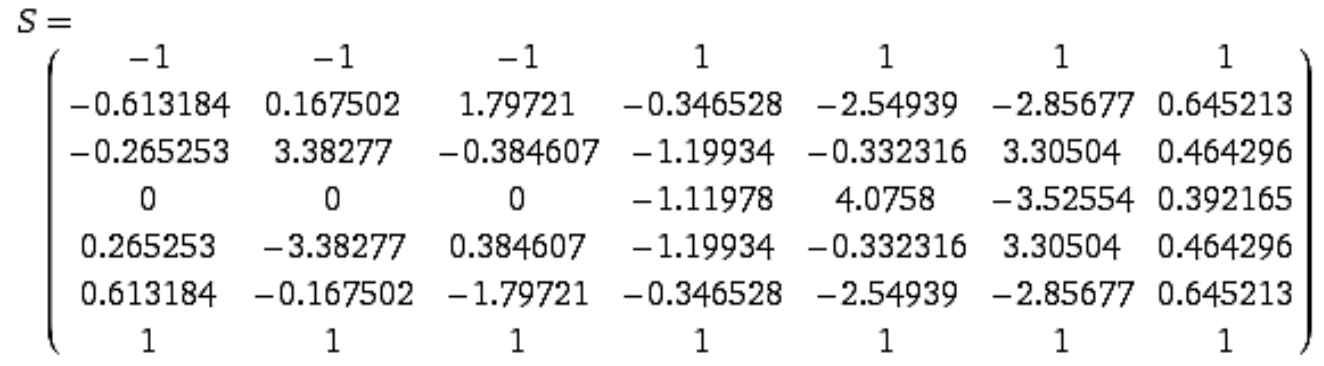

$J=\left(\begin{array}{ccccccc}-177.333 & 0 & 0 & 0 & 0 & 0 & 0 \\ 0 & -10.1218 & 0 & 0 & 0 & 0 & 0 \\ 0 & 0 & -2.54493 & 0 & 0 & 0 & 0 \\ 0 & 0 & 0 & -18.4947 & 0 & 0 & 0 \\ 0 & 0 & 0 & 0 & -10.5847 & 0 & 0 \\ 0 & 0 & 0 & 0 & 0 & 3.52885 & 0 \\ 0 & 0 & 0 & 0 & 0 & 0 & 229.551\end{array}\right)$

$S^{-1}=\left(\begin{array}{ccccccc}-0.345697 & -0.211976 & -0.0916973 & -5.55112 \times 10^{-17} & 0.0916973 & 0.211976 & 0.345697 \\ -0.0400925 & 0.00671559 & 0.135624 & -6.93889 \times 10^{-18} & -0.135624 & -0.00671559 & 0.0400925 \\ -0.114211 & 0.20526 & -0.0439263 & 2.77556 \times 10^{-17} & 0.0439263 & -0.20526 & 0.114211 \\ 0.156964 & -0.0543926 & -0.188253 & -0.175765 & -0.188253 & -0.0543926 & 0.156964 \\ 0.0314151 & -0.0800893 & -0.0104397 & 0.128042 & -0.0104397 & -0.0800893 & 0.0314151 \\ 0.019012 & -0.054313 & 0.0628356 & -0.0670276 & 0.0628356 & -0.054313 & 0.019012 \\ 0.292608 & 0.188795 & 0.135857 & 0.114751 & 0.135857 & 0.188795 & 0.292608\end{array}\right)$ 


\section{Condition number:}

111.705

DiagonalMatrix $[\{2,8,20,28,50,82,126\}]$

Result:

$\left(\begin{array}{ccccccc}2 & 0 & 0 & 0 & 0 & 0 & 0 \\ 0 & 8 & 0 & 0 & 0 & 0 & 0 \\ 0 & 0 & 20 & 0 & 0 & 0 & 0 \\ 0 & 0 & 0 & 28 & 0 & 0 & 0 \\ 0 & 0 & 0 & 0 & 50 & 0 & 0 \\ 0 & 0 & 0 & 0 & 0 & 82 & 0 \\ 0 & 0 & 0 & 0 & 0 & 0 & 126\end{array}\right)$

Dimensions:

\section{7 (rows) $\times 7$ (columns)}

Matrix plot:

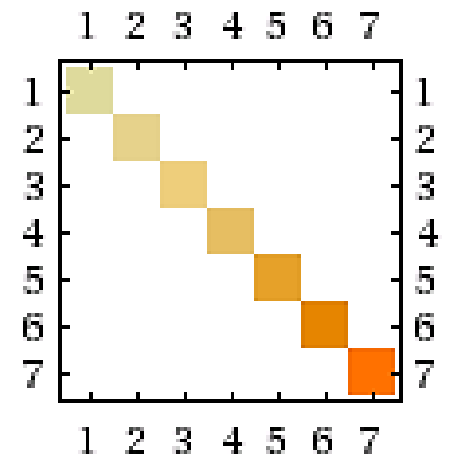

Determinant:

4628736000

Trace:

316

Characteristic polynomial:

$-x^{7}+316 x^{6}-36752 x^{5}+1997872 x^{4}-53507696 x^{3}+679862656 x^{2}-3475467520$ $x+4628736000$ 
Eigenvalues:

$$
\begin{aligned}
& \lambda_{1}=126 \\
& \lambda_{2}=82 \\
& \lambda_{3}=50 \\
& \lambda_{4}=28 \\
& \lambda_{5}=20 \\
& \lambda_{6}=8 \\
& \lambda_{7}=2
\end{aligned}
$$

\section{Diagonalization:}

$$
M=S . J . S^{-1}
$$

\section{where}

$$
\begin{aligned}
M & =\left(\begin{array}{ccccccc}
2 & 0 & 0 & 0 & 0 & 0 & 0 \\
0 & 8 & 0 & 0 & 0 & 0 & 0 \\
0 & 0 & 20 & 0 & 0 & 0 & 0 \\
0 & 0 & 0 & 28 & 0 & 0 & 0 \\
0 & 0 & 0 & 0 & 50 & 0 & 0 \\
0 & 0 & 0 & 0 & 0 & 82 & 0 \\
0 & 0 & 0 & 0 & 0 & 0 & 126
\end{array}\right) \\
S & =\left(\begin{array}{ccccccc}
1 & 0 & 0 & 0 & 0 & 0 & 0 \\
0 & -1 & 0 & 0 & 0 & 0 & 0 \\
0 & 0 & 1 & 0 & 0 & 0 & 0 \\
0 & 0 & 0 & -1 & 0 & 0 & 0 \\
0 & 0 & 0 & 0 & 1 & 0 & 0 \\
0 & 0 & 0 & 0 & 0 & -1 & 0 \\
0 & 0 & 0 & 0 & 0 & 0 & 1
\end{array}\right) \\
J & =\left(\begin{array}{cccccccc}
2 & 0 & 0 & 0 & 0 & 0 & 0 \\
0 & 8 & 0 & 0 & 0 & 0 & 0 \\
0 & 0 & 20 & 0 & 0 & 0 & 0 \\
0 & 0 & 0 & 28 & 0 & 0 & 0 \\
0 & 0 & 0 & 0 & 50 & 0 & 0 \\
0 & 0 & 0 & 0 & 0 & 82 & 0 \\
0 & 0 & 0 & 0 & 0 & 0 & 126
\end{array}\right) \\
S^{-1} & =\left(\begin{array}{ccccccc}
1 & 0 & 0 & 0 & 0 & 0 & 0 \\
0 & -1 & 0 & 0 & 0 & 0 & 0 \\
0 & 0 & 1 & 0 & 0 & 0 & 0 \\
0 & 0 & 0 & -1 & 0 & 0 & 0 \\
0 & 0 & 0 & 0 & 1 & 0 & 0 \\
0 & 0 & 0 & 0 & 0 & -1 & 0 \\
0 & 0 & 0 & 0 & 0 & 0 & 1
\end{array}\right)
\end{aligned}
$$




\section{Condition number:}

$\frac{1}{c} \begin{gathered}\text { Inverse: } \\ 516600 \\ 0\end{gathered}\left(\begin{array}{ccccccc}258300 & 0 & 0 & 0 & 0 & 0 & 0 \\ 0 & 64575 & 0 & 0 & 0 & 0 & 0 \\ 0 & 0 & 25830 & 0 & 0 & 0 & 0 \\ 0 & 0 & 0 & 18450 & 0 & 0 & 0 \\ 0 & 0 & 0 & 0 & 10332 & 0 & 0 \\ 0 & 0 & 0 & 0 & 0 & 6300 & 0 \\ 0 & 0 & 0 & 0 & 0 & 0 & 4100\end{array}\right)$

The tetrahedral numbers from the Pascal Triangle

Magic numbers are twice the tetrahedral numbers $(1,4,10,20,35,56 \ldots)$ from the Pascal Triangle".

Example:

\begin{tabular}{|c||c|c|c|c|c|c|c|c|c|c|}
\hline Row & & & & & & & & & & \\
\hline \hline A & 1 & 1 & 1 & $\mathbf{1}$ & 1 & 1 & 1 & 1 & $\mathbf{1}$ & $\ldots . .1$ \\
\hline B & 1 & 2 & 3 & $\mathbf{4}$ & 5 & 6 & 7 & 8 & 9 & $\ldots . . \mathrm{N}$ \\
\hline C & 1 & 3 & 6 & $\mathbf{1 0}$ & 15 & 21 & 28 & 36 & 45 & $\ldots . \mathrm{N}$ \\
\hline \hline D & 1 & 4 & 10 & $\mathbf{2 0}$ & 35 & 56 & 84 & 120 & 165 & $\ldots . . \mathrm{N}$ \\
\hline $\mathrm{E}$ & 1 & 5 & 15 & $\mathbf{3 5}$ & 70 & 126 & 210 & 330 & 495 & $\ldots . \mathrm{N}$ \\
\hline F & 1 & 6 & 21 & $\mathbf{5 6}$ & 126 & 252 & 462 & 792 & 1287 & $\ldots . \mathrm{N}$ \\
\hline G & 1 & 7 & 28 & $\mathbf{8 4}$ & 210 & 462 & 924 & 1716 & 3003 & $\ldots . . \mathrm{N}$ \\
\hline H & 1 & 8 & 36 & $\mathbf{1 2 0}$ & 330 & 792 & 1716 & 3432 & 6435 & $\ldots . \mathrm{N}$ \\
\hline \hline J & $\mathbf{1}$ & 9 & 45 & 165 & 495 & 1287 & 3003 & 6435 & 12870 & $\ldots . . \mathrm{N}$ \\
\hline &. &. &. &. &. &. &. &. &. &. \\
\hline \hline & $\mathbf{I}$ & $\mathrm{N}$ & $\mathrm{N}$ & $\mathrm{N}$ & $\mathrm{N}$ & $\mathrm{N}$ & $\mathrm{N}$ & $\mathrm{N}$ & $\mathrm{N}$ & $\ldots . \mathrm{N}$ \\
\hline
\end{tabular}

The rows of Pascal's triangle are conventionally enumerated starting with row $n=0$ at the top. The entries in each row are numbered from the left beginning with $k=0$ and are usually staggered relative to the numbers in the adjacent rows. A simple construction of the triangle proceeds in the following manner. On row 0 , write only the number 1 . Then, to construct the elements of following rows, add the number above and to the left with the 
number above and to the right to find the new value. If either the number to the right or left is not present, substitute a zero in its place. For example, the first number in the first row is $0+1=1$, whereas the numbers 1 and 3 in the third row are added to produce the number 4 in the fourth row.
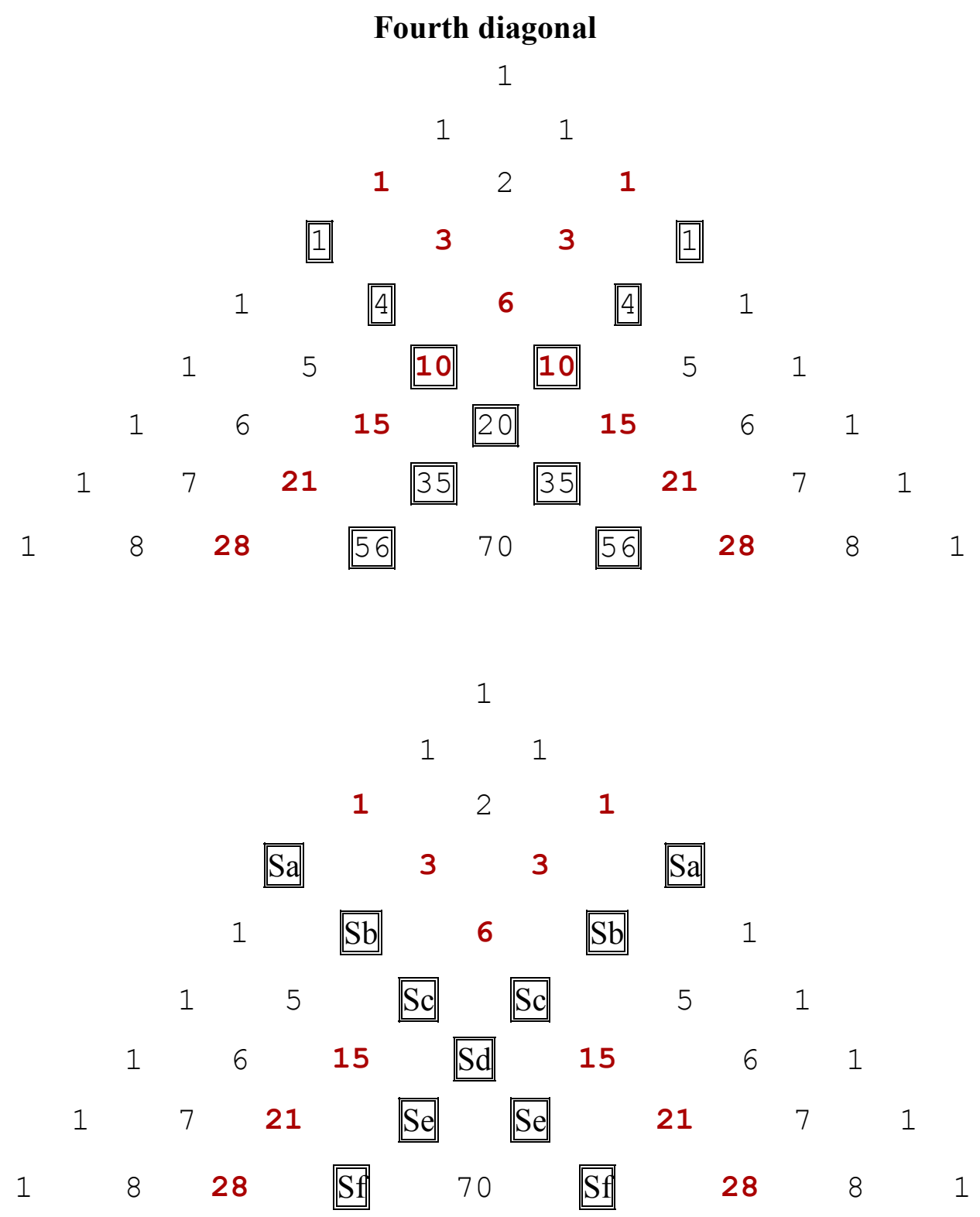

$$
\begin{gathered}
\mathrm{Sa}=\mathrm{S} 1=1 ; \\
\mathrm{Sb}=(\mathrm{S} 1+\mathrm{S} 2)=(1+3)=4 ; \\
\mathrm{Sc}=(\mathrm{S} 1+\mathrm{S} 2+\mathrm{S} 3)=(1+3+6)=10 \\
\mathrm{Sd}=(\mathrm{S} 1+\mathrm{S} 2+\mathrm{S} 3+\mathrm{S} 4)=(1+3+6+10)=20 \\
\mathrm{Se}=(\mathrm{S} 1+\mathrm{S} 2+\mathrm{S} 3+\mathrm{S} 4+\mathrm{S} 5)=(1+3+6+10+15)=35 \\
\mathrm{Sf}=(\mathrm{S} 1+\mathrm{S} 2+\mathrm{S} 3+\mathrm{S} 4+\mathrm{S} 5+\mathrm{S} 6)=(1+3+6+10+15+21)=56 \\
\text { etc. }
\end{gathered}
$$




\section{That expansion looks like}

\begin{tabular}{|c||c|c|}
\hline Diagonal 3 & $\begin{array}{c}\text { Groups numbers } \\
\text { in diagonal 3 }\end{array}$ & Tetrahedral numbers \\
\hline 1 & $1 \rightarrow$ & 4 \\
\hline 3 & $(1+3) \rightarrow$ & 10 \\
\hline \hline 6 & $(1+3+6) \rightarrow$ & 20 \\
\hline \hline 10 & $(1+3+6+6+10) \rightarrow$ & 53 \\
\hline \hline 15 & $(1+3+6+10+15+21+28) \rightarrow$ & 56 \\
\hline \hline 21 & $(1+3+6+10+15+21+28+36) \rightarrow$ & 120 \\
\hline \hline 36 & & 54 \\
\hline \hline
\end{tabular}

etc.

A Pentatope Numbers in the Pascal's triangle were developed with the expansion of the numbers from the diagonal 4:

\section{That expansion looks like}

\begin{tabular}{|c|l|c||}
\hline Diagonal 4 & \multicolumn{1}{|c||}{$\begin{array}{c}\text { Groups numbers } \\
\text { in diagonal } 4\end{array}$} & $\begin{array}{c}\text { Pentatope } \\
\text { numbers }\end{array}$ \\
\hline \hline 1 & $1 \rightarrow$ & 1 \\
\hline \hline 4 & $(1+4) \rightarrow$ & 5 \\
\hline \hline 10 & $(1+4+10) \rightarrow$ & 15 \\
\hline \hline 20 & $(1+4+10+20) \rightarrow$ & 15 \\
\hline \hline 35 & $(1+4+10+20+35) \rightarrow$ & 126 \\
\hline \hline 56 & $(1+4+10+20+35+56) \rightarrow$ & 210 \\
\hline \hline 120 & $(1+4+10+20+35+56+84) \rightarrow$ & 330 \\
\hline
\end{tabular}

etc. 
This construction is related to the binomial coefficients by Pascal's rule, which says that if then for any nonnegative integer $n$ and any integer $k$ between 0 and $n$.

$$
\begin{gathered}
(x+y)^{n}=\sum_{k=0}^{n}\left(\begin{array}{l}
n \\
k
\end{array}\right) x^{n-k} y^{k} \\
\left(\begin{array}{l}
n \\
k
\end{array}\right)=\left(\begin{array}{l}
n-1 \\
k-1
\end{array}\right)+\left(\begin{array}{c}
n-1 \\
k
\end{array}\right) \\
1{ }^{1}{ }^{1}{ }^{1}{ }^{2}{ }^{1} 4^{1} 1 \\
1
\end{gathered}
$$

The first six rows of Pascal's triangle

In particular, the first six shells are:

- $\quad$ level 0: 2 states $(l=0)=2$.

- $\quad$ level 1: 6 states $(l=1)=6$.

- $\quad$ level 2: 2 states $(l=0)+10$ states $(l=2)=12$.

- $\quad$ level 3: 6 states $(l=1)+14$ states $(l=3)=20$.

- level 4: 2 states $(l=0)+10$ states $(l=2)+18$ states $(l=4)=30$.

- $\quad$ level 5: 6 states $(l=1)+14$ states $(l=3)+22$ states $(l=5)=42$.

where for every $l$ there are $2 l+1$ different values of $m_{l}$ and 2 values of $m_{s}$, giving a total of $4 l+2$ states for every specific level.

\begin{tabular}{|c|c|c|c|c|c|c|c|c|c|c|c|c|}
\hline $\begin{array}{c}\text { Row } \\
\text { Number }\end{array}$ & \multicolumn{10}{|c|}{ Binomial Expansion } & Connection & $\begin{array}{c}\text { Code } \\
101\end{array}$ \\
\hline $\mathrm{A}_{0} \quad \hat{\mathrm{A}}$ & $\hat{\mathrm{A}}$ & $\hat{\mathrm{A}}$ & $\hat{\mathrm{A}}$ & $\hat{A}$ & 01 & $\hat{\mathrm{A}}$ & $\hat{A}$ & $\hat{\mathrm{A}}$ & $\hat{A}$ & $\hat{\mathrm{A}}$ & 1 & $101^{0}$ \\
\hline A1 $\hat{A}$ & $\hat{\mathrm{A}}$ & $\hat{\mathrm{A}}$ & $\hat{\mathrm{A}}$ & 01 & $\hat{\mathrm{A}}$ & 01 & $\hat{\mathrm{A}}$ & $\hat{\mathrm{A}}$ & $\hat{A}$ & $\hat{A}$ & 101 & $101^{1}$ \\
\hline $\mathrm{A} 2 \hat{\mathrm{A}}$ & $\hat{\mathrm{A}}$ & $\hat{\mathrm{A}}$ & 01 & $\hat{A}$ & 02 & $\hat{\mathrm{A}}$ & 01 & $\hat{\mathrm{A}}$ & $\hat{A}$ & $\hat{A}$ & 10201 & $101^{2}$ \\
\hline A3 $\hat{A}$ & $\hat{\mathrm{A}}$ & 01 & $\hat{\mathrm{A}}$ & 03 & $\hat{\mathrm{A}}$ & 03 & $\hat{\mathrm{A}}$ & 01 & $\hat{\mathrm{A}}$ & $\hat{\mathrm{A}}$ & 1030301 & $101^{3}$ \\
\hline A4 $\hat{A}$ & 01 & $\hat{\mathrm{A}}$ & 04 & $\hat{\mathrm{A}}$ & 06 & $\hat{\mathrm{A}}$ & 04 & $\hat{\mathrm{A}}$ & 01 & $\hat{A}$ & 104060401 & $101^{4}$ \\
\hline A5 01 & $\hat{\mathrm{A}}$ & 05 & $\hat{A}$ & 10 & $\hat{A}$ & 10 & $\hat{A}$ & 05 & $\hat{A}$ & 01 & 10510100501 & $101^{5}$ \\
\hline etc. & ." & $"$ & " & " & " & $"$ & $"$ & $"$ & $"$ & $"$ & etc. & \\
\hline
\end{tabular}

\section{Example:}

A binomial is a polynomial expression with two terms, such as:

These numbers are twice the values of triangular numbers from the Pascal Triangle: $1,3,6,10,15,21 \ldots$ 


\section{Example :}

\begin{tabular}{|c|c|c|c|c|c|c|c|c|c|c|}
\hline \multicolumn{11}{|c|}{ Row } \\
\hline A & 1 & 1 & 1 & 1 & 1 & 1 & 1 & 1 & 1 & $\ldots . .1$ \\
\hline B & 1 & 2 & 3 & 4 & 5 & 6 & 7 & 8 & 9 & $\ldots . . . \mathrm{N}$ \\
\hline $\mathrm{C}$ & 1 & 3 & 6 & 10 & 15 & 21 & 28 & 36 & 45 & $\ldots . . . \mathrm{N}$ \\
\hline $\mathrm{D}$ & 1 & 4 & 10 & 20 & 35 & 56 & 84 & 120 & 165 & $\ldots . . \mathrm{N}$ \\
\hline $\mathrm{E}$ & 1 & 5 & 15 & 35 & 70 & 126 & 210 & 330 & 495 & ...... N \\
\hline $\mathrm{F}$ & 1 & 6 & 21 & 56 & 126 & 252 & 462 & 792 & 1287 & $\ldots . . \mathrm{N}$ \\
\hline $\mathrm{G}$ & 1 & 7 & 28 & 84 & 210 & 462 & 924 & 1716 & 3003 & ...... N \\
\hline $\mathrm{H}$ & 1 & 8 & 36 & 120 & 330 & 792 & 1716 & 3432 & 6435 & ...... N \\
\hline $\mathrm{J}$ & 1 & 9 & 45 & 165 & 495 & 1287 & 3003 & 6435 & 12870 & $\ldots . . . \mathrm{N}$ \\
\hline & 1 & N & $\dot{\mathrm{N}}$ & $\dot{\mathrm{N}}$ & $\dot{\mathrm{N}}$ & $\dot{\mathrm{N}}$ & $\dot{\mathrm{N}}$ & $\dot{\mathrm{N}}$ & $\dot{\mathrm{N}}$ & $\dot{\mathrm{N}}$ \\
\hline
\end{tabular}

1

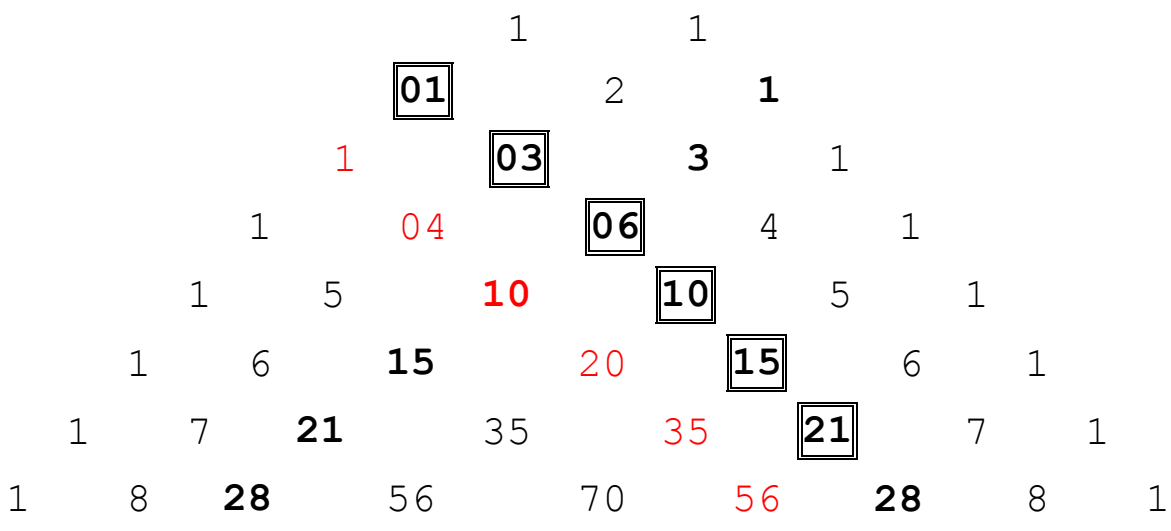

INPUT $=$ Diagonal 1,03,06,10,15,21;

OUTPUT $=$ Diagonal 1,04,10,20,35,56;

\section{TRIANGULAR NUMBER}

A triangular number or triangle number numbers the objects that can form an equilateral triangle, as in the diagram on the right. The $n$th triangle number is the number of dots in a triangle with $n$ dots on a side; it is the sum of the $n$ natural numbers from 1 to $n$. The sequence of triangular numbers (sequence A000217 inOEIS), starting at the 0th triangular number, is: $0,1,3,6,10,15,21,28,36,45,55, \ldots$ 


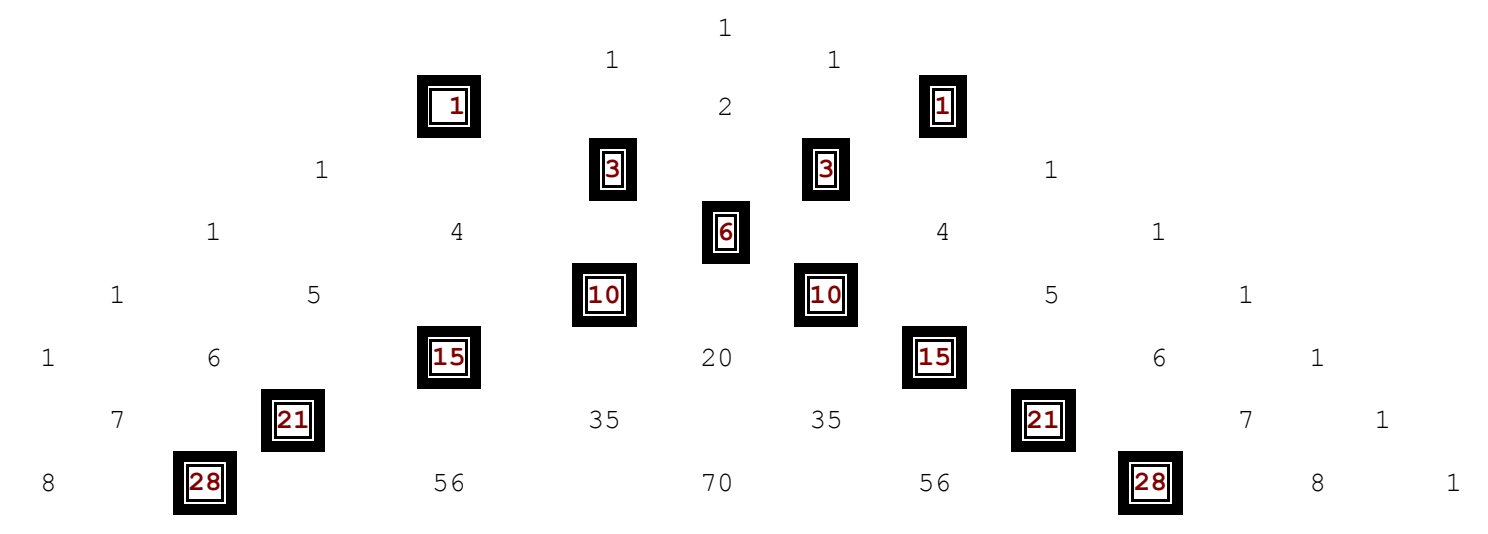

Triangular numbers in the Pascal's triangle were developed with the expansion of the numbers from the diagonal 2:

That expansion looks like:

\begin{tabular}{|c|c|c|}
\hline Diagonal 2 & $\begin{array}{c}\text { Groups numbers } \\
\text { in diagonal } 2\end{array}$ & $\begin{array}{c}\text { Triangular } \\
\text { numbers }\end{array}$ \\
\hline 1 & $1 \rightarrow$ & 1 \\
\hline 2 & $(1+2) \rightarrow$ & 3 \\
\hline 3 & $(1+2+3) \rightarrow$ & 6 \\
\hline 4 & $(1+2+3+4) \rightarrow$ & 10 \\
\hline 5 & $(1+2+3+4+5) \rightarrow$ & 15 \\
\hline 6 & $(1+2+3+4+5+6) \rightarrow$ & 21 \\
\hline 7 & $(1+2+3+4+5+6+7) \rightarrow$ & 28 \\
\hline 8 & $(1+2+3+4+5+6+7+8) \rightarrow$ & 36 \\
\hline & etc. & \\
\hline
\end{tabular}

Groups numbers

in the triangular numbers 


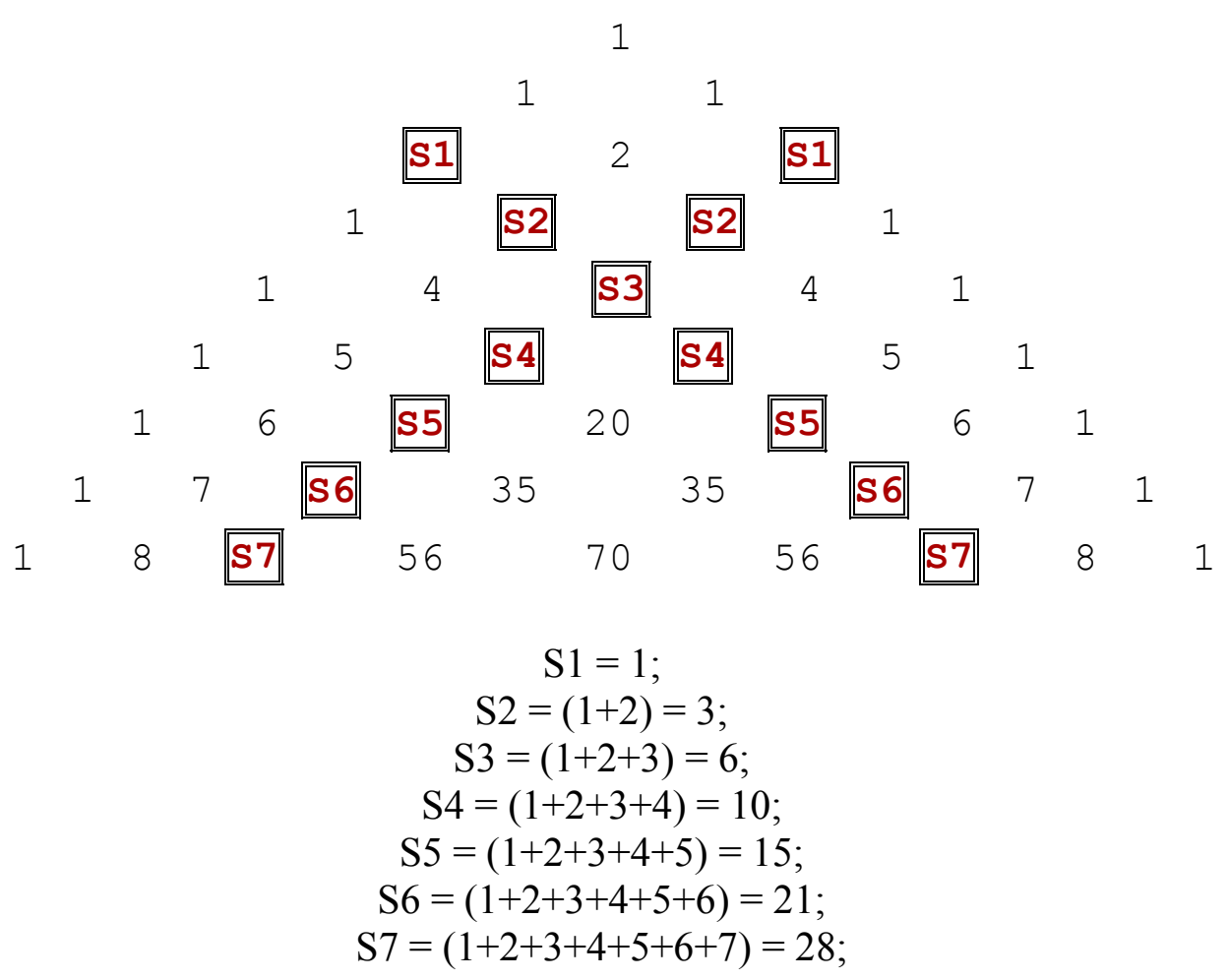

Another way to predict magic (and semi-magic) numbers is by laying out the idealized filling order (with spin-orbit splitting but energy levels not overlapping). For consistency s is split into $\mathrm{j}=1 / 2$ and $\mathrm{j}=-1 / 2$ components with 2 and 0 members respectively. Taking leftmost and rightmost total counts within sequences marked bounded by / here gives the magic and semi-magic numbers.

- $\mathrm{s}(2,0) / \mathrm{p}(4,2)>2,2 / 6,8$, so (semi)magic numbers $2,2 / 6,8$

- $\mathrm{d}(6,4): \mathrm{s}(2,0) / \mathrm{f}(8,6): \mathrm{p}(4,2)>14,18: 20,20 / 28,34: 38,40$, so $14,20 / 28,40$

- $\mathrm{g}(10,8): \mathrm{d}(6,4): \mathrm{s}(2,0) / \mathrm{h}(12,10): \mathrm{f}(8,6): \mathrm{p}(4,2)>50,58,64,68,70,70 / 82,92,100,106,110,112$, so $50,70 / 82,112$

- $\quad \mathrm{i}(14,12): \mathrm{g}(10,8): \mathrm{d}(6,4): \mathrm{s}(2,0) / \mathrm{j}(16,14): \mathrm{h}(12,10): \mathrm{f}(8,6): \mathrm{p}(4,2)>$

$126,138,148,156,162,166,168,168 / 184,198,210,220,228,234,238,240$, so $126,168 / 184,240$

The rightmost predicted magic numbers of each pair within the quartets bisected by / are double tetrahedral numbers from the Pascal Triangle: 2,8,20,40,70,112,168,240 are $2 \mathrm{x}$ $1,4,10,20,35,56,84,120 \ldots$, and the leftmost members of the pairs differ from the rightmost by double triangular numbers: $2-2=0,8-6=2,20-14=6,40-28=12,70-50=20,112-82=30$, $168-126=42,240-184=56$, where $0,2,6,12,20,30,42,56 \ldots$ are $2 \mathrm{x} 0,1,3,6,10,15,21,28 \ldots$. http://en.wikipedia.org/wiki/Nuclear_shell_model..

\section{CONCLUSIONS}

These digital pictures reveal to us a whole new dimension to Digital nuclear shell model. They reveal to us that the cosmic process is strictly conditioned and determined by 
programmatic, cybernetic and information principles. Now we have the exact scientific proofs that there is a Standard Model of Elementary Particles language that can be described by the theory of systems and cybernetics, and which functions in accordance with certain principles.

\section{References}

[1] L. Kurić, International Letters of Chemistry, Physics and Astronomy 10 (2014) 62-73.

[2] L. Kurić, J. Comput Sci Biol 2 (2009) 101-116.

[3] L. Kurić, International Letters of Chemistry, Physics and Astronomy 13(1) (2014) 42-53.

[4] L. Kurić, Journal de la Societe de statistique de Paris 127(2) (1986).

[5] L. Kurić, GJMR 10(1) (2010) 15.

[6] L. Kurić, Advances and Applications in Bioinformatics and Chemistry (2010) 45-58.

[7] L. Kurić, GJMR 1(1) (2010) 15.

[8] L. Kurić, International Journal of Computer Technology and Application 2(2) (2011) 216-241.

[9] L. Kurić, International Journal of Computer Technology and Application 2(2) (2011) 258-273.

[10] L. Kurić, Journal of Chemical Engineering and Material Science 2(5) (2011).

[11] L. Kurić, International Letters of Chemistry, Physics and Astronomy 11(3) (2014) 202-213.

[12] L. Kurić, International Letters of Chemistry, Physics and Astronomy 12 (2014) 31-50.

[13] L. Kurić, International Letters of Chemistry, Physics and Astronomy 13(1) (2014) $11-20$.

[14] Lutvo Kurić, International Letters of Chemistry, Physics and Astronomy 13(1) (2014) $42-53$. 